\title{
A real-time interactive stereo display platform for 3D medical images
}

\author{
Bing Chen, Xiao-Jian Zhang, Wang-Lin Ke, Lu-Lu Xie and Jun-Hai Wen ${ }^{\dagger}$, \\ Department of Biomedical Engineering, School of Life Science, \\ Beijing Institute of Technology, Beijing, China. \\ †Email: wenjh@bit.edu.cn
}

\begin{abstract}
In recent years, three-dimensional display technology has developed rapidly, and its applications in medical, scientific research and games are very attractive. The research and development of magnetic resonance imaging(MRI), computed tomography(CT), ultrasonography(US) and other medical imaging equipment provide a great deal of medical anatomical information, and the $3 \mathrm{D}$ visualizations of those medical images are the important application of virtual reality technology. In this paper, we built a real-time interactive stereo display platform for 3D medical images using OpenGL interface based on MFC framework. The platform achieved interactive operations: such as rotation, translation, scaling and cutting of 3D model.
\end{abstract}

Keywords: Stereoscopic display; Medical images; Real-time interactive.

\section{Introduction}

With the development of science, technology and the improvement of living standards, people pay more attention to the medical problem. The threedimensional display of medical images of image processing and computer graphics is a hot research in recent years. The three-dimensional visualization of medical images is an important research content of scientific visualization. The research and development of magnetic resonance imaging(MRI), computed tomography(CT), ultrasonography(US) and other medical imaging equipment provide a great deal of medical anatomical information, and the 3D visualizations of those medical images are the important application of virtual reality technology[1].

Human vision is the result of interaction between eyes and brain [2]. When a person is observing an object, it can be coordinated by two eyes to gain depth perception. The images projected on the retina are different since the spatial positions of two eyes are different [3]. The left eye can see the left side more and the right eye can get more information from the right side, and the subtle differences between the left and right eye's observation is the binocular parallax that people of-ten called[4]. The formation of eye's depth perception comes 
from monocular cues and binocular cues [5]. Due to some geometric features of the object itself, such as the shadow of the object, and life experiences, even when the observer is looking with one eye or looking at flat two-dimensional images, it allow us to form some kind of stereoscopic perception. However, good stereo vision only can be obtained when observing with two eyes.

At present, the stereoscopic display device has been developed a variety of products, including stereo glasses, stereo display, stereo projection equipment, and the most common stereo imaging technology is based on the principle of binocular parallax. Based on the principle of binocular parallax, the threedimensional display technology is mainly divided into glasses / helmet-mounted displays (HMD) and grating stereoscopic display [6], a helmet-mounted display directly provide the left and right images for the corresponding eye on two screens [7], in stereo glasses display, the monocular image of left eye or right eye is displayed on the same screen, and left eye and right eye only see its own image by glasses. Based on the display rules, the stereo glasses can be divided into: Red and blue 3D stereo glasses, the LCD shutter glasses and 3D polarized glasses [8].

Recently, many research institutes and most hospitals have realized the necessity of medical digitalization. They've made a lot of investment, and they have developed their own visualization system [9]. However, the commercialization of medical visualization system is still in a primary stage. Meanwhile, in the stereoscopic display technology, China was focused on some theoretical research in the past few years. In this paper, we achieved a real-time three-dimensional stereoscopic display platform, which can carry out volume rendering and three-dimensional display of 3D volume data. This platform use wireless LCD shutter glasses as hardware support, and have high degree of freedom, interactivity and strong sense of immersion.

\section{Implementation}

\subsection{The development environment and hardware support}

In this study, we used Dell Workstation with Intel(R)Xeon(R) 3.50GHZ CPU, 8GB RAM. Our development platform is Microsoft Visual Studio 2005, and we take advantage of CUDA (compute unified device architecture) and GPU, which can make the program super high performance, and make the real-time interaction possible. At the same time, we use OpenGL, the professional graphics interface, which defines cross-programming language and crossplatform programming interface.

We chose NVIDIA 3D Vision stereo display device as hardware support. 3D Vision is a kind of 3D stereo display technology of NVIDIA, equipped with 
the corresponding 3D glasses, USB IR emitter. In the entire display process, the active shutter glasses need to switch on and off in a high speed so that the monitor needs to support high refresh rate, which at least up to $120 \mathrm{~Hz}$. In this work, the ASUS VG 278HE LED monitor was used, which resolution is $1920 * 1080$ and the refresh rate can reach $144 \mathrm{~Hz}$. Besides, it supports active shutter glasses and can be compatible with NVIDIA stereo vision.

\subsection{Volume Rendering and the generation of stereo image pair}

In general, there are two main ways to get volume data: one is medical data that reflects the body's organs, which obtained through CT, MRI and other medical devices. Another one is obtained through scientific calculation, such as finite element analysis and numerical simulation of volume data. In this paper, the data we used is raw data, which the long by width by height is $256 \times 256 \times 256$.

The rendering methods can be divided into surface rendering method and volume rendering method [10]. The volume rendering method performs well on display quality, but the rendering speed is usually slow because of very large amount of computation. However, in recent years, the rapid development of computer hardware makes it possible to speed up the rendering speed. For the sake of final rendering result and the display quality, in this paper, we used the ray casting method, which is a kind of the volume rendering method, to render the three-dimensional volume data directly. In order to optimize the performance of the program and accelerate the rendering speed of ray casting rendering, in this study, we take advantage of the GPU hardware features, using CUDA framework and OpenGL to complete the rendering and displaying of 3D volume data.

There are two approaches to get the stereo image pair: using the stereo camera to take pictures or video to get the stereo image pair, or generating the stereo image pair from the three-dimensional model by the computer algorithm. In medicine, we have to use the second approach to obtain the stereo image pair of the internal body from the CT/MRI volume data.

Since OpenGL [11] defined a three-dimensional view frustum, we can obtain an image through a view frustum. In order to imitate the stereo parallax and generate stereo image pair, we can apply the coordinate transform in a view frustum to simulate the human binocular parallax. The original view frustum facing the target, move the view frustum to the left and to the right in a parallel line with the same distance, thus we can get two frustums. These two view frustums can imitate human eyes to "look" at the object, just like human eyes are watching the object.

We set the current viewpoint as the centric viewpoint, after that the left viewpoint and right viewpoint can be generated through the transformation from 
the centric viewpoint, which must have the same field of view, and then the stereo image pair with parallax can be generated by rendering respectively. Literature [12] proposed a method for the calculation of the coordinate Transformation: We establish a PE-centered coordinates system, supposing the mono eye's coordinates is $P_{e}\left(X_{e}, Y_{e}, Z_{e}\right)$, the distance between two eyes is e, the horizontal rotation angle is $\theta$, and vertical rotation angle is $\varphi$. The left and right eye coordinates $P_{e l}\left(X_{e l}, Y_{e l}, Z_{e l}\right)$ and $P_{e r}\left(X_{e r}, Y_{e r}, Z_{e r}\right)$ can be calculated from the formulas below:

$$
\begin{aligned}
& x_{e l}=x_{e}+\frac{e \cos (\theta+\pi / 2)}{2}, y_{e l}=y_{e}, z_{e l}=z_{e}+\frac{e \sin (\theta+\pi / 2)}{2} \\
& x_{e r}=x_{e}+\frac{e \cos (\theta-\pi / 2)}{2}, y_{e r}=y_{e}, z_{e r}=z_{e}+\frac{e \sin (\theta-\pi / 2)}{2}
\end{aligned}
$$

After obtained the parameters of the right and left viewpoint through calculation, we can use the OpenGL interface to render the images respectively, then we get the stereo image pairs based on $3 \mathrm{D}$ volume data model, as shown in Fig. 1.

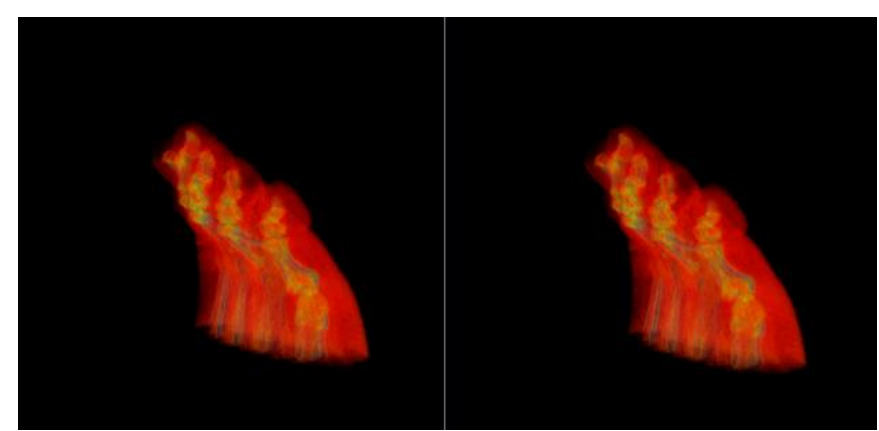

Fig. 1. The stereo image pair.

\subsection{Implementation of the stereo display platform for $3 D$ medical images}

In this section, we will show how to implement the stereoscopic display. The specific process is as follows:

(1)Install 3D vision driver and the USB IR emitter driver; finish the $3 \mathrm{D}$ vision test wizard settings, and open the 3D vision; the refresh rate of monitor should be adjusted to $120 \mathrm{~Hz}$.

(2)Create a window using MFC platform, assign the display area and design widgets for interactive operations.

(3)Load the 3D volume data and initialize the display parameters and viewpoint; initialize the display mode and enable the stereo display. 
(4)Calculate the left viewpoint and right viewpoint from the current viewpoint using the formula (2.1) and formula (2.2). The left image was obtained by using the left viewpoint's parameters, and we directly write the left image into the back buffer GL_BACK_LEFT; and then the right image was obtained by using the right viewpoint's parameters, and was written to the back buffer GL_BACK_RIGHT. Hence the left image and right image can be alternatively rendered and shown on the screen, and the active shutter glasses are synchronized by the USB IR emitter while the monitor is refreshing.

(5)Build the callback functions for the widgets on the panel according to the message mechanism of MFC frame, which achieve interactive operations.

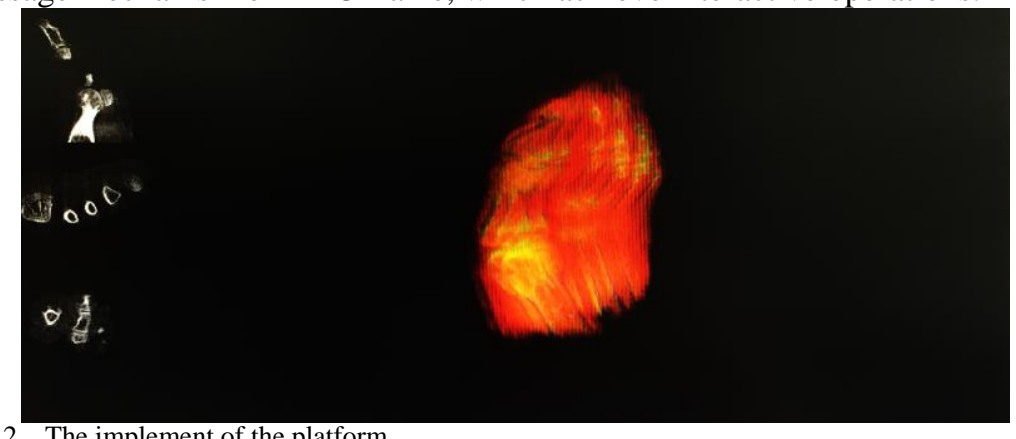

Fig. 2. The implement of the platform.

Our software platform achieved the stereo display of the 3D medical data, this study selected NVIDIA active shutter glasses as hardware support, which ensure the display quality. The platform also achieved the mouse-based interactive operation, such as rotation, translation, scaling and cutting of 3D model. The stereo display platform is shown in Fig. 2.

\section{Conclusion and Future Work}

This study built a real-time interactive stereo display platform for $3 \mathrm{D}$ medical images using OpenGL interface based on MFC framework. The platform achieved the interactive operations: adjust the dis-play parameter according to the user's requirements, separate the bones and soft tissues and choose the region of interest to display.

In this work, the interactive operation is the mouse-based operation; the next work we will focus on is the improvement of interactivity. The force feedback data glove will be used to control the 3D medical model directly, such as grasping, moving, rotating and stripping action, which will provide users with more realistic and natural $3 \mathrm{D}$ interaction experience. 


\section{Acknowledgements}

This work was supported in part by the National Natural Science Foundation of China ( No.31170955), and in part by the National key re-search program of China (2016YFC0105104).

\section{References}

1. Ying Juan. Development and application of three-dimensional medical image visualization technology[J].Medical Equipment, 2007, (09): 20-22.

2. Ming-li Wang. Parallax stereoscopic image and its algorithm[M].Beijing: Science Press, 2005.

3. Shi K, Wang F. The development of stereoscopic display technology[J]. 2010, 4:V4-276 - V4-280.

4. Shin-ichi Uehara, Hiroyasu Ujike, Goro Hamagishi, et al. Standardization based on human factors for 3D display: performance characteristics and measurement methods[J]. SPIE-IS\&T Electronic Imaging, 2009: 7524, 752407-1.

5. Yao-lin Tan. Image Information System Principle [M] Beijing: Tsinghua University Press, 2006.12-117, 206-214.

6. Wang Qionghua, Wang Ai-hong. Three-dimensional Display Review [J] Journal of Computer Applications, 2010,03: 579-581 + 588 .

7. Zheng Hua-dong, Yu Ying-jiel, Cheng Wei-mingo A review on three dimensional display techniques[J].OpticaI Technique, 2008(3)

8. Zhang Yinan; He Jing; Yuan Jie "A stereoscopic display system based on a volumetric image model", Natural Computation (ICNC), 2013 Ninth International Conference on, On page(s): 1273 - 1277.

9. Cao X, Huang $\mathrm{H} \mathrm{K}$. Current status and future advances of digital radiography and PACS[J]. IEEE Engineering in Medicine \& Biology Magazine the Quarterly Magazine of the Engineering in Medicine \& Biology Society, 2000, 19(5):80-88.

10. Miao Lin. Information Processing and body three-dimensional data field rendering [D]. National University of Defense Technology, 2003.

11. OpenGL Architecture Review Board, Dave Sheiner, Mason Woo, Jackie Neider, Tom Davis. OpenGL Programming Guide: The Official Guide to Learning OpenGL, Version 2.1, Sixth Edition[M]. Pearson Education, 2009.

12. Liu Haiyan, Cheng Lei, Wang Yan. A practical HMD stereoscopic image generation technology [J] Shenyang University of Technology, 2005, (03): 43-46. 